\title{
Sidestream Dark Field Imaging of the Microcirculation to Assess Preeclampsia Microvascular Dysfunction
}

\author{
Amelie Pelland $^{\mathrm{a}}$, Ronald B. George ${ }^{\mathrm{a}, \mathrm{e}}$, Christian Lehmann ${ }^{\mathrm{a}, \mathrm{b}, \mathrm{c}}$, Jillian Coolen ${ }^{\mathrm{d}}$
}

\begin{abstract}
Background: Development of predictive models of preeclampsia has only yielded modest results. We hypothesized that impaired indices of microcirculatory function could be detected using sidestream dark field imaging. The objective of this study was to examine microvascular function in women at high risk for preeclampsia at midgestation.
\end{abstract}

Methods: Women between 16 and 22 weeks of gestation were screened for eligibility. Patients were recruited if they met eligibility criteria indicating high risk for preeclampsia. Investigators performed non-invasive sidestream dark field imaging of the sublingual microcirculation. Images were analyzed to determine microcirculatory parameters (microvascular flow index, perfused vessel density, total vessel density, and proportion of perfused vessels). After delivery, charts were reviewed to determine if they developed gestational hypertension, preeclampsia or severe preeclampsia.

Results: Twelve of 66 participants (18.2\%) developed preeclampsia or severe preeclampsia during the course of their pregnancy. Microvascular flow index was not significantly different between participants with normal pregnancies and participants with preeclampsia or severe preeclampsia $(2.75 \pm 0.38$ vs. $2.80 \pm 0.34$, respectively; $\mathrm{P}=0.459$ ). Similarly, there were no significant differences between groups in the remaining microcirculatory parameters.

Conclusions: Sidestream dark field imaging of the sublingual microcirculation may remain an appropriate tool to identify women at risk for preeclampsia, albeit later in pregnancy.

Keywords: Pregnancy; Preeclampsia; Microcirculation; Diagnostic imaging

Manuscript submitted January 26, 2018, accepted February 23, 2018

aDepartment of Anesthesiology, Pain Management, and Perioperative Medicine, Dalhousie University, Halifax, NS, Canada

${ }^{b}$ Department of Pharmacology, Dalhousie University, Halifax, NS, Canada

'Department of Microbiology and Immunology, Dalhousie University, Halifax, NS, Canada

dDepartment of Obstetrics and Gynecology, Dalhousie University, Halifax, NS, Canada

${ }^{\mathrm{e}}$ Corresponding Author: Ronald B. George, IWK Health Centre, Department of Women's and Obstetric Anesthesia, 5850/5980 University Avenue, P.O. Box 9700, Halifax, NS B3K 6R8, Canada. Email: rbgeorge@dal.ca

doi: https://doi.org/10.14740/jocmr3368w

\section{Introduction}

Preeclampsia is a multi-system hypertensive disorder of pregnancy that is a significant cause of maternal morbidity and mortality worldwide [1]. The pathophysiology of preeclampsia is incompletely understood, but a growing body of evidence suggests that poor placentation as a result of abnormal cytotrophoblast invasion of uterine spiral arteries is an inciting event [2]. Placental ischemia leads to the systemic release of anti-angiogenic factors causing widespread maternal endothelial activation and dysfunction $[3,4]$. Recent clinical research has shown that anti-angiogenic signaling and vascular abnormalities manifest prior to the development of clinical signs and symptoms, even as early as mid-gestation $[5,6]$. As of yet, efforts to develop models for the prediction of preeclampsia have only yielded modest results; modelling accuracy has not approached that required for clinical use [7].

Functional monitoring of microcirculatory perfusion has been well established in other disease states characterized by endothelial vascular dysfunction, such as sepsis [8]. Oxygenation, nutrient exchange and elimination of waste products necessary for adequate cellular metabolism occur at the level of the microcirculation, which is defined as the grouping of vessels with a diameter smaller than $100 \mu \mathrm{m}$. Less is known about the microvascular consequences of pregnancy and pregnancy-related pathology. Peripheral microcirculatory flow, as well as small vessel density is reduced in women with preeclampsia $[9,10]$. Peripheral capillary density was also found to be decreased as early as 20 weeks of gestation in women who would later develop preeclampsia, and capillary rarefaction at 27 to 32 weeks of gestation was shown to be an independent predictor for the development of the disease [11].

Sidestream dark field (SDF) imaging produces high-contrast real-time video images of the human microcirculation facilitating computer-assisted offline analyses of microvascular blood flow with high inter- and intra-observer reliability [12]. It permits quantification of microvascular blood flow using the microvascular flow index (MFI) and perfused vessel density (PVD), which may be an important tool in assessing vascular dysfunction in pregnancy and preeclampsia. This study is a prospective observational examination of microvascular function in women at high risk for preeclampsia at mid-gestation. It was hypothesized that indices of microcirculatory function as measured by SDF imaging would be impaired prior to the appearance of clinical disease in women with pregnancies com- 


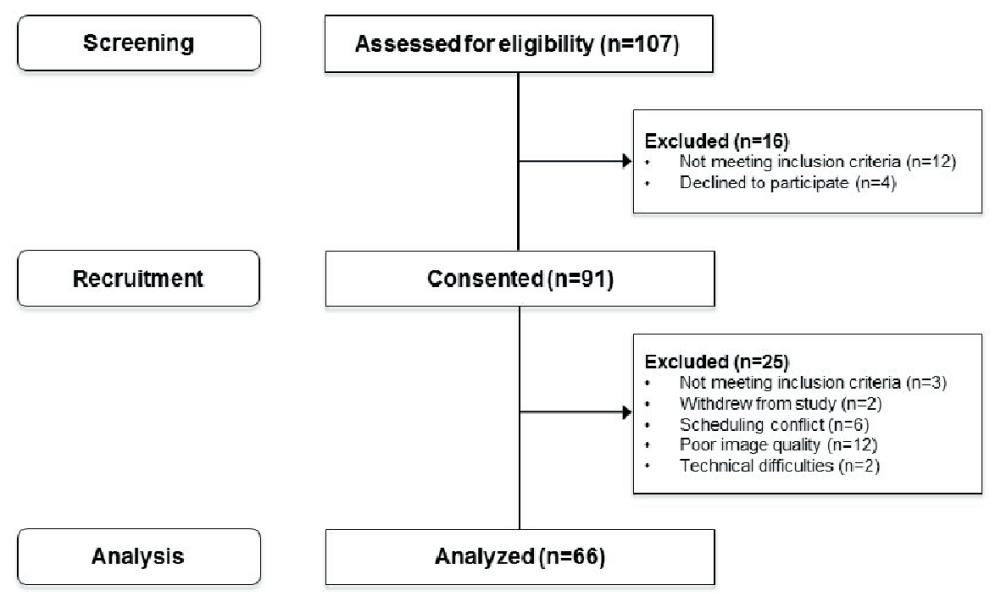

Figure 1. Flow diagram of patient screening, recruitment and analysis from September 2014 to July 2016.

plicated by preeclampsia.

\section{Methods}

The study was conducted from September 2014 to July 2016 at the IWK Health Centre, Halifax, NS, Canada. The study protocol was approved by the institutional Research Ethics Board (IWK REB \# 1016039). Written informed consent was obtained from all participants meeting inclusion criteria.

Women presenting for a prenatal clinic visit at the IWK Health Centre prior to 22 weeks of gestation were screened for eligibility. Patients at high risk for preeclampsia were included as participants if they met at least one of the following criteria: preeclampsia in a previous pregnancy, pre-existing renal disease, pre-existing diabetes mellitus, antiphospholipid syndrome, obesity with a body mass index $(\mathrm{BMI}) \geq 35$, pre-existing hypertension, or both maternal age $>40$ years and family history of preeclampsia in a first degree relative. These inclusion criteria are risk factors for preeclampsia as defined by The Society of Obstetricians and Gynaecologists of Canada's clinical practice guidelines regarding hypertensive disorders of pregnancy [13]. Participants were excluded if they were current smokers, had consumed caffeine within $6 \mathrm{~h}$ of imaging or were non-English speaking and reading. Age, parity, gestational age, and BMI were recorded for each participant at the time of SDF imaging.

Study investigators performed all analytical non-invasive SDF measurements (MicroScan, MicroVision Medical, Amsterdam, Netherlands). Imaging was completed during a single clinic visit when participants were between 16 and 22 weeks of gestation. After measuring oral temperature and blood pressure, imaging of the sublingual microcirculation was performed as per standards outlined by De Backer et al [12] in the semi-recumbent position. The SDF device equipped with a sterile probe cap was applied to the sublingual mucosa after gentle cleansing with isotonic saline. After manually optimizing image focus, steady images of at least $20 \mathrm{~s}$ in duration were obtained, with care taken to avoid pressure artefacts. Digital recordings of five different visual fields were obtained for each participant, with minimal intervals between recordings. Blood pressure measurements were repeated following SDF imaging.

Video images, which contained no identifiable information, were analyzed blindly by a single investigator following randomization. A separate investigator not involved with video analysis completed the randomization with a computer-generated randomization table. The SDF images were reviewed to determine the microcirculatory parameters (MFI, PVD, total vessel density (TVD), proportion of perfused vessels (PPV) using the Automated Vascular Analysis (AVA 3.0 ${ }^{\circledR}$ ) software (MicroVision Medical, Amsterdam, Netherlands) [12].

After delivery, participants' charts were reviewed to determine if they developed gestational hypertension, preeclampsia or severe preeclampsia. Gestational hypertension, preeclampsia and severe preeclampsia were defined according to The Society of Obstetricians and Gynaecologists of Canada's clinical practice guidelines regarding hypertensive disorders of pregnancy [13]. Briefly, gestational hypertension was defined by new-onset hypertension after 20 weeks of gestation with systolic blood pressure $\geq 140 \mathrm{~mm} \mathrm{Hg}$ and/or diastolic blood pressure $\geq 90 \mathrm{~mm} \mathrm{Hg}$. Preeclampsia was defined as hypertension accompanied by proteinuria or one or more of a list of adverse conditions, while severe preeclampsia was characterized as preeclampsia with onset prior to 34 weeks of gestation with heavy proteinuria or one or more adverse conditions. Adverse conditions reflect end-organ damage as manifested by maternal symptoms and signs, laboratory abnormalities and/ or evidence of fetal morbidity. The adverse conditions include, but are not limited to headache or visual symptoms, elevated creatinine, thrombocytopenia, right upper quadrant or epigastric pain, and chest pain or dyspnea [13].

A power analysis was performed for the difference between two independent means of MFI using our recently completed assessment of maternal microcirculation [14]. Calculations were made using $G^{*}$ Power version 3.0.10 (G*Power, University of Dusseldorf) with a standard deviation of 0.5 and an effect size of 1.0 (12.5\% difference in MFI), with alpha of 0.05 and power of 0.8 . Given the overall incidence of preec- 
Table 1. Participant Characteristics at the Time of Sidestream Dark Field Imaging

\begin{tabular}{llll}
\hline Characteristic & No preeclampsia $(\mathbf{n}=\mathbf{5 4})$ & Preeclampsia $(\mathbf{n}=\mathbf{1 0})$ & Severe preeclampsia $(\mathbf{n}=\mathbf{2})$ \\
\hline Age (years) & $31.7 \pm 3.9$ & $31.0 \pm 4.1$ & $32.5 \pm 5.0$ \\
BMI $\left(\mathrm{kg} / \mathrm{m}^{2}\right)$ & $31.4 \pm 9.8$ & $32.4 \pm 8.2$ & $35.1 \pm 1.0$ \\
Gestational age (weeks) & $19.2 \pm 1.7$ & $19.8 \pm 1.7$ & $19.3 \pm 0.6$ \\
Inclusion criteria (n (\%)) & & & 0 \\
$\quad$ Previous preeclampsia & $11(20.4)$ & $4(40)$ & 0 \\
Pre-existing renal disease & $2(3.7)$ & $1(10)$ & $1(50)$ \\
Pre-existing diabetes mellitus & $18(33.3)$ & $1(10)$ & 0 \\
Antiphospholipid syndrome & $1(1.9)$ & $1(10)$ & $1(50)$ \\
BMI $\geq 35$ & $29(53.7)$ & $3(30)$ & 0 \\
Pre-existing hypertension & $5(9.3)$ & $2(20)$ & $1(50)$ \\
Multiple pregnancy & $4(7.4)$ & $4(40)$ & 0 \\
Age $>40$ years & $3(5.6)$ & 0 & 0 \\
Family history of preeclampsia & $1(1.9)$ & 0 & 0 \\
\hline
\end{tabular}

*Several patients were enrolled who met more than one inclusion criterion. Data are presented as $\mathrm{n}(\%)$ or mean \pm standard deviation (SD).

lampsia of 2-3\% and a 10-fold higher likelihood of developing preeclampsia in our high-risk cohort, it was estimated that 20 $30 \%$ of our participants would develop preeclampsia [15]. To accommodate the allocation of non-preeclamptic participants to preeclamptic participants (allocation ratio $0.25-0.45$ ), it was determined that 50 participants would be required. To account for loss to follow up, technical issues, etc., the sample size was increased by $50 \%$, with a goal to enroll 75 patients as study participants.

Statistical analysis was performed with SPSS 20.0 (SPSS Inv., Chicago, IL, USA). Data are presented as mean \pm standard deviation (SD). Variables were compared with the Student's $t$ test. The primary outcome was the difference in MFI between the normal participants and participants with preeclampsia.

\section{Results}

During the study period, a total of 107 patients were assessed for eligibility and consent was obtained from 91 of those patients (Fig. 1). A further 25 patients were excluded from analysis for a variety of reasons, the most common of which was technical issues and problems with poor image quality on review (presence of secretions, inadequate focus, motion artefacts [10]). A total of 66 patients were included in the final analysis. Eleven participants were taking $81 \mathrm{mg}$ ASA. Of those, three were diagnosed with preeclampsia.

Demographic characteristics are presented in Table 1. Twelve of the participants $(18.2 \%)$ developed preeclampsia or severe preeclampsia during the course of their pregnancy. Age, BMI and gestational age at the time of SDF imaging were similar between participants with normal pregnancies and those with preeclampsia. Median gravidity and parity were 3 and 1 for the no preeclampsia and preeclampsia groups. Both participants who developed severe preeclampsia were primigravidae. Neither blood pressure nor temperature at the time of imaging was different between groups (not shown). Obesity was a common risk factor for inclusion across all groups, representing over $50 \%$ of participants with no preeclampsia. Previous preeclampsia and pre-existing diabetes mellitus were also common risk factors in this same group, while other risk factors were each present in less than $10 \%$ of participants. Previous personal history of preeclampsia and multiple pregnancy were the most common risk factors present in patients with preeclampsia, as each was present in $40 \%$ of patients in this group.

MFI was not significantly different between participants with normal pregnancies and participants with preeclampsia or severe preeclampsia $(2.75 \pm 0.38$ vs. $2.80 \pm 0.34$, respectively; $\mathrm{P}=0.459$ ) (Fig. 2a). Similarly, there were no significant differences in TVD, PVD and PPV between the two groups (Fig. 2b-d).

A second analysis separating the participants who developed severe preeclampsia from those with non-severe preeclampsia also showed that there were no significant differences in microcirculation parameters between these groups (Table 2). Participants with severe preeclampsia had similar MFI values as participants with non-severe preeclampsia and no preeclampsia $(2.79 \pm 0.34$ vs. $2.79 \pm 0.34$ vs. $2.75 \pm 0.38$, respectively). There was, however, a trend toward an increased PPV with increasing disease severity.

\section{Discussion}

This study demonstrates that sublingual microcirculatory indices (MFI, TVD, PVD, and PPV) as measured by SDF imaging are similar between patients who develop preeclampsia and those who do not in a high-risk group between 16 and 22 weeks of gestation. This is in contrast with reports of decreased peripheral capillary density measured by different methods later in pregnancy in women with established preeclampsia $[9,10,16]$. One study using SDF, however, found that only 


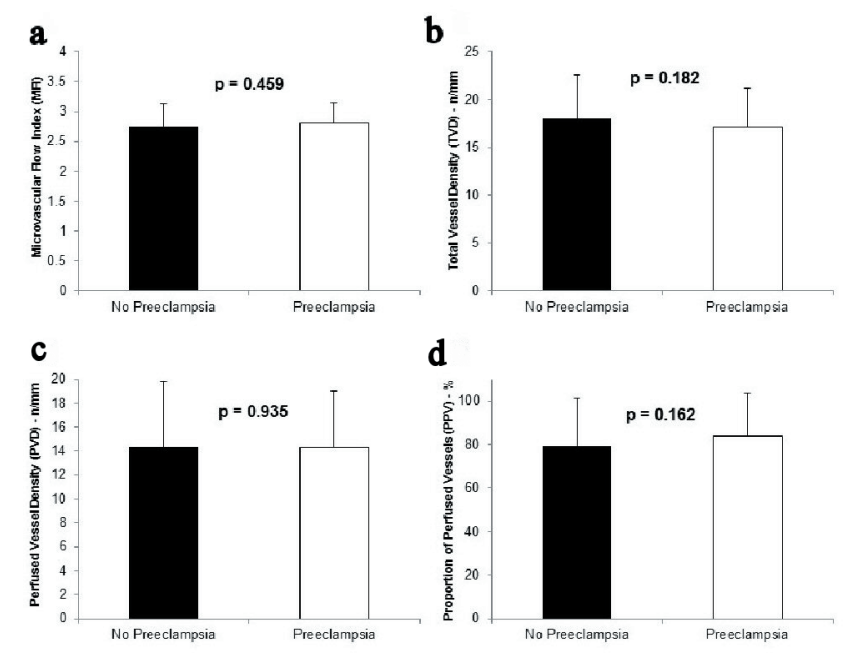

Figure 2. MFI (a), TVD (b), PVD (c), and PPV (d) in participants who did not develop preeclampsia $(n=54)$ and those who developed preeclampsia $(n=12)$. The participants who developed severe preeclampsia are included in the preeclampsia group.

women with HELLP syndrome demonstrated impaired microcirculation compared with healthy women and women with preeclampsia [17]. This study is the first to describe microcirculatory function early in the second trimester of pregnancy; Antonios et al demonstrated capillary rarefaction between 20 and 24 weeks of gestation by skin capillaroscopy [11].

Placentation becomes fully established between 12 and 16 weeks of gestation and although conflicting about the exact chronology of increasing level of circulating anti-angiogenic factors, studies reveal that it is unlikely that these levels increase before 16 - 17 weeks of gestation [18]. The temporal relationship between the rise in anti-angiogenic factors and microcirculatory dysfunction is further unknown. One report, investigating endothelial function using acetylcholine and sodium nitroprusside iontophoresis, demonstrated increased vascular reactivity starting at 22 weeks of gestation during pregnancies in which preeclampsia would develop [19]; however, SDF imaging does not measure vascular reactivity, but functional capillary density, and as such the investigators were expecting to detect earlier changes. There are several factors that may have contributed to the lack of measurable differences: 1) Higher resolution imaging may be required for more accurate measurement of microvascular blood flow. A new generation of imaging microscopes using incident dark field imaging (IDF) have been shown to produce higher resolution images
[20], and may be capable of detecting the expected changes. 2) If these changes are more subtle than expected, the statistical power analysis would need to be adjusted and more participants may be required to discern a statistically significant difference in microcirculatory parameters between the groups. 3) A more objective, automated image analysis software might be required to reduce human error in the quantification of microvascular blood flow. One such software package exists, made by Braedius Medical B.V. (Huizen, The Netherlands), and may refine data analysis enough to detect a difference.

The major strength of this study lies in the definition of the inclusion criteria. These criteria allowed the recruitment of a group of patients with a 10-fold increased incidence of preeclampsia as expected. Although not powered to detect a difference in participants having developed severe disease, this study was otherwise adequately powered for the primary outcome. The MFI values measured in this study were also consistent with values previously measured in full term pregnancies by our group [14]. It is not known if this value changes over the course of pregnancy, but this lends a degree of validity to our results.

One limitation of this study was the lack of inclusion of a group of participants with healthy pregnancies for comparison. While it can be argued that the participants who did not develop preeclampsia formed an adequate control group, each participant had at least one risk factor for vascular disease

Table 2. : Microcirculation Indices in Participants With no Preeclampsia, Preeclampsia, and Severe Preeclampsia

\begin{tabular}{llll} 
Microcirculatory Parameter & No preeclampsia $(\mathbf{n}=\mathbf{5 4})$ & Preeclampsia $(\mathbf{n}=\mathbf{1 0})$ & Severe preeclampsia $(\mathbf{n}=\mathbf{2})$ \\
\hline MFI & $2.75 \pm 0.38$ & $2.79 \pm 0.34$ & $2.79 \pm 0.34$ \\
TVD $(\mathrm{n} / \mathrm{mm})$ & $17.99 \pm 4.62$ & $17.12 \pm 4.12$ & $17.14 \pm 3.62$ \\
PVD $(\mathrm{n} / \mathrm{mm})$ & $14.32 \pm 5.47$ & $14.06 \pm 4.82$ & $15.23 \pm 4.43$ \\
PPV $(\%)$ & $79.38 \pm 21.38$ & $82.86 \pm 21.10$ & $87.76 \pm 13.07$ \\
\hline
\end{tabular}

Data are presented as means $\pm \mathrm{SD}$. 
by virtue of the inclusion criteria. Women likely to develop metabolic syndrome, in particular, are heavily represented in this sample. Of note, nine of the $54(16.7 \%)$ patients without preeclampsia did receive a diagnosis of gestational hypertension during their pregnancies. It is unclear if gestational hypertension arises from the same mechanisms as preeclampsia, but this may further indicate that the control group participants were not free of vascular disease. The second limitation to the study was the number of participants excluded due to poor imaging quality, which was mostly because of inadequate focus and motion artifact. This may represent a form of selection bias if impaired microcirculation is inherently difficult to image. Improved software for image analysis may mitigate this problem in the future.

\section{Conclusions}

In conclusion, we did not detect a functional difference in microcirculation between women who will develop preeclampsia and those who will not. SDF imaging of the sublingual microcirculation may remain an appropriate modality to identify women at risk for the disease, albeit later in pregnancy. Defining the microvascular effects of current preventive therapy, such as aspirin, and microcirculation-targeted therapy may also be an interesting application of SDF imaging in pregnancy in the future.

\section{Disclosure}

This work was supported by a Category A grant from the IWK Health Centre, and a Dalhousie University Department of Anesthesia, Pain Management and Perioperative Medicine Internal Project Grant. The authors have no conflict of interest to disclose.

\section{References}

1. Ghulmiyyah L, Sibai B. Maternal mortality from preeclampsia/eclampsia. Semin Perinatol. 2012;36(1):56-59.

2. Fisher SJ. Why is placentation abnormal in preeclampsia? Am J Obstet Gynecol. 2015;213(4 Suppl):S115-122.

3. Palei AC, Spradley FT, Warrington JP, George EM, Granger JP. Pathophysiology of hypertension in pre-eclampsia: a lesson in integrative physiology. Acta Physiol (Oxf). 2013;208(3):224-233.

4. Wang A, Rana S, Karumanchi SA. Preeclampsia: the role of angiogenic factors in its pathogenesis. Physiology (Bethesda). 2009;24:147-158.

5. Levine RJ, Lam C, Qian C, Yu KF, Maynard SE, Sachs BP, Sibai BM, et al. Soluble endoglin and other circulating antiangiogenic factors in preeclampsia. N Engl J Med. 2006;355(10):992-1005.

6. Noori M, Donald AE, Angelakopoulou A, Hingorani AD, Williams DJ. Prospective study of placental angiogenic factors and maternal vascular function before and after preeclampsia and gestational hypertension. Circulation.
2010;122(5):478-487.

7. Rana S, Powe CE, Salahuddin S, Verlohren S, Perschel $\mathrm{FH}$, Levine RJ, Lim KH, et al. Angiogenic factors and the risk of adverse outcomes in women with suspected preeclampsia. Circulation. 2012;125(7):911-919.

8. De Backer D, Ospina-Tascon G, Salgado D, Favory R, Creteur J, Vincent JL. Monitoring the microcirculation in the critically ill patient: current methods and future approaches. Intensive Care Med. 2010;36(11):1813-1825.

9. Anim-Nyame N, Sooranna SR, Johnson MR, Gamble J, Steer PJ. A longitudinal study of resting peripheral blood flow in normal pregnancy and pregnancies complicated by chronic hypertension and pre-eclampsia. Cardiovasc Res. 2001;50(3):603-609.

10. Hasan KM, Manyonda IT, Ng FS, Singer DR, Antonios TF. Skin capillary density changes in normal pregnancy and pre-eclampsia. J Hypertens. 2002;20(12):2439-2443.

11. Antonios TF, Nama V, Wang D, Manyonda IT. Microvascular remodelling in preeclampsia: quantifying capillary rarefaction accurately and independently predicts preeclampsia. Am J Hypertens. 2013;26(9):1162-1169.

12. De Backer D, Hollenberg S, Boerma C, Goedhart P, Buchele G, Ospina-Tascon G, Dobbe I, et al. How to evaluate the microcirculation: report of a round table conference. Crit Care. 2007;11(5):R101.

13. Magee LA, Pels A, Helewa M, Rey E, von Dadelszen P, Canadian Hypertensive Disorders of Pregnancy Working G. Diagnosis, evaluation, and management of the hypertensive disorders of pregnancy. Pregnancy Hypertens. 2014;4(2):105-145.

14. George RB, Munro A, Abdo I, McKeen DM, Lehmann C. An observational assessment of the sublingual microcirculation of pregnant and non-pregnant women. Int J Obstet Anesth. 2014;23(1):23-28.

15. Pare E, Parry S, McElrath TF, Pucci D, Newton A, Lim KH. Clinical risk factors for preeclampsia in the 21 st century. Obstet Gynecol. 2014;124(4):763-770.

16. Anim-Nyame N, Sooranna SR, Johnson MR, Gamble J, Steer PJ. Resting peripheral blood flow in normal pregnancy and in pre-eclampsia. Clin Sci (Lond). 2000;99(6):505-510.

17. Cornette J, Herzog E, Buijs EA, Duvekot JJ, Rizopoulos D, Hop WC, Tibboel D, et al. Microcirculation in women with severe pre-eclampsia and HELLP syndrome: a casecontrol study. BJOG. 2014;121(3):363-370.

18. Rana S, Karumanchi SA, Levine RJ, Venkatesha S, RauhHain JA, Tamez H, Thadhani R. Sequential changes in antiangiogenic factors in early pregnancy and risk of developing preeclampsia. Hypertension. 2007;50(1):137142.

19. Khan F, Belch JJ, MacLeod M, Mires G. Changes in endothelial function precede the clinical disease in women in whom preeclampsia develops. Hypertension. 2005;46(5):1123-1128.

20. van Elteren HA, Ince C, Tibboel D, Reiss IK, de Jonge $\mathrm{RC}$. Cutaneous microcirculation in preterm neonates: comparison between sidestream dark field (SDF) and incident dark field (IDF) imaging. J Clin Monit Comput. 2015;29(5):543-548. 